\title{
Management strategies for unintentional dural puncture: a Canadian experience survey in an academic setting
}

\author{
Samantha Russell, MBCHB, FRCPC $\mathbb{D}$
}

Received: 5 October 2017/Revised: 19 December 2017/Accepted: 21 December 2017/Published online: 11 January 2018

(c) Canadian Anesthesiologists' Society 2018

\section{To the Editor,}

Unintentional dural puncture (UDP) is a complication of epidural anesthesia with reported rates of $0.5-4 \% .^{1}$ It is accompanied by acute and chronic morbidity and, most notably, post-dural puncture headache (PDPH). Techniques and interventions employed to reduce the incidence and treat PDPH have varying levels of success. ${ }^{1}$

We conducted a cross-sectional survey to learn about common practices for managing obstetrical UDP in Winnipeg, Canada. Following ethics approval (University of Manitoba, HS20027 H2016:311), anesthesiologists assigned to all maternity units were identified and then surveyed between January 2015 and October 2016 using SurveyMonkey ${ }^{\mathrm{TM}}$ (SurveyMonkey Inc., San Mateo, CA, USA). Data collected included the anesthesiologists' clinical (personal and supervising) experience in obstetric anesthesia, epidural insertion technique, UDP, and PDPH management. Responses were reported using descriptive statistics.

Responses were received from 62 of 102 anesthesiologists (61\%). Mean (standard deviation) anesthesia experience was 13 (9) yr. Anesthesiologists reported using a $17 \mathrm{G}(n=48,77 \%)$ Tuohy needle $(n=51,82 \%)$ via a midline $(n=62,100 \%)$ sitting approach $(n=61,98 \%)$ and advanced the needle with the bevel oriented cephalad ( $n=60,97 \%)$, using a loss of resistance (LOR) to air technique to identify the epidural space $(n=47,76 \%)$.

Participants were asked what percentage of UDP was managed in their practice with the following techniques.

S. Russell, MBCHB, FRCPC ( $\square)$

Department of Anesthesia, Perioperative and Pain Medicine, St

Boniface Hospital, Winnipeg, MB, Canada

e-mail: samanthalrussell@gmail.com
Respondents $(n=60)$ reported re-siting the epidural needle (64\%), threading the epidural catheter into the subarachnoid space (30\%), and abandoning the procedure for alternative labour analgesia (6\%). Respondents who stated they placed spinal catheters $(n=44)$ reported they were removed immediately post-delivery $(n=28,64 \%)$ or retained in situ for $\geq 24 \mathrm{hr}(n=16,36 \%)$. No prophylactic epidural blood patch (EBP) was performed by the survey group.

In response to the question "What is the ideal time following delivery to perform an EBP for PDPH treatment?" $79 \%(n=46)$ of anesthesiologists responded $\geq 24 \mathrm{hr}$, unless there was coexisting cranial nerve involvement that prompted earlier intervention $(n=31$, $54 \%)$. Anesthesiologists reported reviewing the last charted vital signs, excluding fever $(n=53,91 \%)$, and a complete blood count $(n=19,33 \%)$ prior to performing the EBP. Intravenous access was obtained by $74 \%(n=43)$ of respondents, but neither a fluid bolus nor medication (anxiolytic, antiemetic, and/or analgesic) was routinely given. Fifty-five percent $(n=32)$ stated they would attempt the EBP at the same vertebral level as the UDP, employing either a sitting $(n=33,57 \%)$ or lateral position $(n=25,43 \%)$. Seventy-two percent $(n=42)$ reported a target $20 \mathrm{~mL}$ EBP volume but would stop injecting if back or neck pain occurred. Eighty-nine percent $(n=52)$ instructed the patient to lay supine for up to two hours post-EBP.

A second EBP was offered by $72 \%(n=42)$ of respondents if the first patch failed and was timed to occur between $24-48 \mathrm{hr}$ after the initial EBP $(n=31)$. The majority $(n=33)$ would proceed without radiologic imaging.

Our respondents' practice reflects the ambiguity of the literature regarding UDP and PDPH management. 
Changing recommendations regarding the LOR to saline or air technique ${ }^{1}$ and a move to siting an intrathecal catheter $^{2,3}$ in the literature were echoed in by our respondents. Local practice is consistent with expert opinion to wait $24 \mathrm{hr}$ to perform a therapeutic EBP. ${ }^{4,5}$ The 20-mL target blood volume is well supported, although some recommend larger volumes to counteract the destabilizing effect of cerebrospinal fluid on the blood clot (patch). ${ }^{4}$

Obstetric UDP is a known risk in epidural analgesia; however, comprehensive evidence-based best practice for management is not available. The diversity of practice and evidence regarding UDP and PDPH suggests that consensus on management is lacking and represents an opportunity for future research.

Acknowledgements This work was funded by the University of Manitoba Department of Anesthesia, Perioperative and Pain Medicine academic oversight committee with statistical analysis by Loring Chuchmach from the George \& Fay Yee Centre for Healthcare Innovation. Neither organization had any role in the design or conduct of this study. Dr. Judith Littleford served as a mentor on this project.
Conflicts of interest None declared.

Editorial responsibility This submission was handled by Dr. Gregory L. Bryson, Deputy Editor-in-Chief, Canadian Journal of Anesthesia.

\section{References}

1. Sachs A, Smiley $R$. Post-dural puncture headache: the worst common complication in obstetric anesthesia. Semin Perinatol 2014; 38: 386-94.

2. Berger CW, Crosby ET, Grodecki W. North American survey of the management of dural puncture occurring during labour epidural analgesia. Can J Anaesth 1998; 45: 110-4.

3. Van de Velde $M$. Accidental dural puncture and postdural puncture headache: what are our options? Reg Anesth Pain Med 2016; 41: 121-2.

4. Scavone BM. Timing of epidural blood patch: clearing up the confusion. Anaesthesia 2015; 70: 119-21.

5. Loeser EA, Hill GE, Bennett GM, Sederberg JH. Time vs. success rate for epidural blood patch. Anesthesiology 1978; 2: 147-8. 